\title{
La théologie dans le schème pascalien des " raisons des effets "
}

\section{Hélène Bouchilloux}

\section{(2) OpenEdition}

1 Journals

\section{Édition électronique}

URL : http://journals.openedition.org/ccibp/550

DOI : $10.4000 /$ ccibp. 550

ISSN : 2493-7460

Éditeur

Centre international Blaise Pascal

\section{Édition imprimée}

Date de publication : 19 octobre 1999

Pagination : $31-38$

ISSN : 0249-6674

\section{Référence électronique}

Hélène Bouchilloux, « La théologie dans le schème pascalien des « raisons des effets » », Courrier du

Centre international Blaise Pascal [En ligne], 20 | 1999, mis en ligne le 06 janvier 2016, consulté le 21 avril 2019. URL : http://journals.openedition.org/ccibp/550 ; DOI : 10.4000/ccibp.550

Ce document a été généré automatiquement le 21 avril 2019.

Centre international Blaise Pascal 


\title{
La théologie dans le schème pascalien des " raisons des effets "
}

\author{
Hélène Bouchilloux
}

1 Les commentateurs de Pascal ont remarqué que la théologie, qui constitue, dans l' Entretien avec M. de Sacy, le point à partir duquel on articule en les jugeant les deux positions philosophiques à la fois antagonistes et solidaires d'Épictète et de Montaigne, fait elle-même l'objet, dans les Écrits sur la grâce, d'une schématisation analogue, puisque c'est à partir de la position théologique augustinienne qu'on articule et qu'on juge désormais pareillement les deux positions théologiques à la fois antagonistes et solidaires des molinistes et des protestants. Ce redoublement ne laisse pas de poser un problème : car, si la philosophie ne peut espérer résoudre les apories auxquelles la condamne la nécessaire unilatéralité de son point de vue sur l'homme que par un passage au point de vue supérieur de la théologie qu'elle doit d'abord accréditer afin de s'y soumettre, n'estce pas encore elle qui intervient quand il s'agit de rattacher la vérité de la théologie à son principe fondamental et d'y comprendre ainsi les erreurs par rapport auxquelles cette vérité doit se définir? La théologie pascalienne est philosophique. N'y a-t-il pas là, cependant, un cercle ? Comment la théologie peut-elle être la vérité de la philosophie et la philosophie, en un autre sens, la vérité de la théologie?

Dans l'Entretien, M. de Sacy présente l'orthodoxie augustinienne comme une vérité à laquelle il convient de se rendre immédiatement et sans détour, alors que Pascal, comme on le sait, la justifie par la médiation et le détour des erreurs philosophiques. Mais l'orthodoxie nue ne suffit peut-être pas plus, au sein de la théologie, que la pure et simple déférence à la théologie, préférée aux méandres de la philosophie. Le contenu vrai de la philosophie est inséparable de la forme vraie par laquelle celle-ci, conjuguant deux vérités, devient capable, dans les Écrits sur la grâce, d'identifier et de révoquer deux erreurs symétriques. Les hérésies, en effet, méconnaissent la dualité de la vérité chrétienne, comme les philosophies méconnaissent la dualité de la nature humaine. La théologie, qui est la vérité de la philosophie, n'est donc pas elle-même vraie hors de la philosophie qui marque en elle le lieu de la vérité, la vérité dans la vérité. 
Or cette réduplication du contenu vrai dans la forme vraie est le nerf du schème des « raisons des effets ». Pour apprécier un discours, on ne doit pas s'en tenir à ce qui est dit, on doit rapporter ce qui est dit aux raisons qui font qu'on le dit. Le peuple, les habiles et les chrétiens parfaits sont d'accord pour dire qu'il faut obéir aux lois, mais en désaccord quant à la raison qui soutient cette obéissance : le peuple veut qu'on y obéisse parce qu'il les croit justes; les habiles, parce qu'ils savent l'homme injuste, et les lois garantes de l'ordre, en tant que lois; les chrétiens parfaits, parce qu'ils savent l'homme à la fois capable et incapable de justice, et l'assujettissent aux lois, garantes de l'ordre en tant que lois, tout à fait justes. Le schème des «raisons des effets» est caractérisé par la disjonction du contenu et de la forme, le point haut étant caractérisé, au contraire, par la réversibilité du contenu et de la forme : le contenu n'y est vrai que soutenu en vérité. Ainsi, la vérité ne consiste pas seulement à dire vrai, mais encore à appréhender le lieu de la vérité. Le peuple et les habiles sont dans le vrai, mais sans voir où se trouve le vrai, sans apercevoir le vrai dans le vrai.

4 Le fait que la théologie s'avère la lumière à partir de laquelle on peut juger n'implique cependant pas qu'elle soit elle-même l'instance du jugement, le jugement consistant dans l'application de ces lumières conformément au schème projectif des " raisons des effets ". Il est important de noter que la théologie, qui est éclairante, est également éclairée. Car, si la perception du vrai dans le vrai opérée par le schème des "raisons des effets" légitime l'obéissance dans le domaine politique en circonscrivant un ordre propre du politique, de sorte que le point de vue de la théologie permet d'assigner la politique à son ordre propre, rien n'interdit l'extension de ce modèle à la théologie elle-même. Certes, contrairement à l'ordre civil qui est sans fondement - il n'est fondé que sur la force et l'imagination ou la concupiscence -, l'ordre religieux est très bien fondé en Jésus-Christ et dans la tradition de l'Église apostolique, mais la question est de savoir si le fondement de l'ordre religieux suffit pour fonder l'obéissance à cet ordre. Ne faut-il pas en outre, pour que l'obéissance soit fondée, que soit circonscrit l'ordre propre du théologique comme il a fallu circonscrire l'ordre propre du politique pour que l'obéissance civile à un ordre civil sans fondement soit elle-même fondée ? En théologie comme en politique, lieu de vérité désigne le lieu de la soumission, son repérage empêchant qu'une soumission aveugle ne cautionne une autorité tyrannique. La soumission n'exclut pas le jugement, où se décide la raison de la soumission.

5 On repartira de la liasse V des Pensées, intitulée "raisons des effets». On montrera comment le point de vue de la théologie commande la fondation de l'obéissance civile à un ordre civil pourtant sans fondement et comment cette fondation de l'obéissance civile s'accompagne de la circonscription de l'ordre propre du politique. On étudiera ensuite le fonctionnement du schème des « raisons des effets » dans l'Entretien avec M. de Sacy, puis dans les Écrits sur la grâce, en insistant sur la rémanence de l'acte philosophique jusque dans la soumission de la philosophie à la théologie, puisqu'il est nécessaire d'appliquer les lumières que procure la théologie, y compris à la théologie elle-même. On décèlera enfin l'ultime portée du schème des « raisons des effets » dans les textes où Pascal assigne la théologie à son ordre propre. Car, si la primauté accordée à la théologie s'explique par la place qu'elle occupe dans le schème des "raisons des effets", elle ne fonde que formellement la vérité qu'elle redresse et elle ne saurait par conséquent se subordonner sans contradiction le contenu des ordres qu'elle ordonne. Pour être réputée ordre supérieur aux autres ordres, la théologie n'en reste pas moins un ordre parmi les autres ordres. Son primat, loin d'abolir la diversité des ordres, la justifie. C'est pourquoi le 
primat conféré à la théologie est précisément ce qui amène à dénoncer la tyrannie de l'autorité religieuse, notamment lorsque celle-ci prétend légiférer dans le domaine politique ou dans le domaine scientifique.

6 C'est dans la liasse V des Pensées qu'est élaborée la notion de « raisons de effets ». Il faut sans doute rappeler tout d'abord, pour en comprendre le sens, que cette expression se rencontre fréquemment dans les traités du graveur Abraham Bosse, celui-ci opposant à l'imitation à vue celle qui est réglée par la perspective géométrique dérivant de la géométrie projective du mathématicien Girard Desargues ${ }^{1}$. Pour peindre correctement un sujet, il ne suffit pas d'en copier extérieurement les apparences, encore faut-il recourir à ce qui n'apparaît nullement mais qui rend raison de ce qui apparaît, à savoir les règles de la représentation. Le schème des "raisons des effets " suppose ainsi la disjonction de l'intérieur et de l'extérieur, le point de vue du peintre converti aux règles de son $\operatorname{art}^{2}$ consistant à appréhender les effets selon les règles de la perspective qui, seules, assurent leur reproduction aussi bien que leur composition picturales. Par ailleurs, la connaissance de ces règles révèle également les défauts des tableaux qui n'obéissent pas à ces règles, de sorte qu'on devient capable de faire la part du bon et du mauvais dans tous les tableaux. Il y a donc une grande différence entre juger selon l'œil (extérieurement) et juger selon les règles (intérieurement).

7 Pascal applique la notion forgée par Abraham Bosse, afin de rendre compte des effets visuels, aux effets anthropologiques et moraux. $\mathrm{Y}$ a-t-il un point indivisible à la lumière duquel ordonner les effets anthropologiques et moraux, comme il en existe un en peinture pour ordonner les effets visuels, ou bien en sommes-nous réduits à la variété des points de vue et à l'absence de toute règle? Telle est la question que posent Lafuma 21, 697 et 699. Or Lafuma 199 montre que c'est précisément en voulant se constituer centre de perspective, non dans l'ordre phénoménal et scientifique mais dans l'ordre essentiel et moral, que l'homme, croyant pouvoir fixer les phénomènes et la science sans se contenter de ne percevoir et de ne connaître jamais qu'un milieu des choses, voit tout son objet s'effondrer dans la double infinité. Le point de vue cherché est donc extra-humain. Ce qui passe la connaissance scientifique des phénomènes nous surpasse. Il $\mathrm{y} a$, certes, un point indivisible en morale comme en peinture, mais ce point relève de la théologie qui parvient seule à articuler les effets anthropologiques et moraux dont elle rend raison jusque dans leur contrariété. Sa place dans la nature n'étant plus définie par sa dépendance à l'égard de Dieu qui en est le centre, l'homme s'efforce de la redéfinir à partir de lui-même. Cependant, l'ignorance de la dualité de sa nature actuelle, dont seule la théologie peut l'instruire, le voue à la fluctuation pendulaire des mouvements contraires : toujours en excès ou en défaut, l'homme privé des lumières et de la théologie ne sait plus à quel rang se mettre ${ }^{3}$. La théologie, qui permet de juger les effets anthropologiques et moraux selon la dualité de la nature humaine, occupe donc le point haut du schème des « raisons des effets » dans la liasse $\mathrm{V}$ des Pensées.

Le point de vue de la théologie met fin à l'oscillation - de l'excès et du défaut répercutée dans celle du pour et du contre ${ }^{4}$ - en renversant absolument chaque effet dans son contraire : ainsi, la revendication d'une pure justice, qui est l'apanage du peuple, n'est satisfaite que retournée en son contraire, dans la reconnaissance de l'injustice surnaturelle des hommes, qui est l'apanage des chrétiens parfaits. En assujettissant les hommes pécheurs à l'ordre légal, Dieu fait justice de leur injustice ${ }^{5}$, et c'est pourquoi, s'il faut obéir à des lois qui ne coïncident jamais avec la pure justice, ce n'est pas d'après le point de vue intermédiaire des habiles, c'est-à-dire parce qu'elles sont lois et que la 
faiblesse factuelle des hommes les condamne à l'ordre à défaut de justice, mais c'est parce que le repli sur l'ordre à défaut de justice n'est justifiable, en tant que peine, que si la faiblesse des hommes, loin d'être simplement factuelle, est rapportée à son véritable principe ou à la dualité enveloppée dans le péché.

Il est impossible, dans ces conditions, de confondre le schème projectif des « raisons des effets ", appliqué à l'anthropologie et à la morale, avec le schème physique des causes des phénomènes ${ }^{6}$. La physique n'a pas affaire à la dualité de l'homme ni à la contrariété des effets qui le caractérise, elle n'a affaire qu'à l'infinité des phénomènes se découvrant progressivement à son investigation. Il s'agit, pour le physicien, de discriminer parmi les hypothèses possibles imaginées pour rendre compte des phénomènes, quelle est la vraie, ce à quoi il ne peut parvenir par la voie positive de la confirmation expérimentale, ce qui requiert, au contraire, le passage par la voie négative de l'infirmation expérimentale : est vérifiée l'hypothèse dont la contradiction est infirmée ${ }^{7}$. Cependant, les hypothèses explicatives se développant à mesure que les phénomènes deviennent plus complexes avec l'amélioration des moyens d'investigation, cette opération donne lieu à un processus de rectification indéfini ${ }^{8}$. Il n'en va pas de même pour l'homme. Car, dans le cas de l'homme, les hypothèses explicatives sont réductibles à l'antagonisme des partisans de sa grandeur et des partisans de sa faiblesse. On peut bien user de l'infirmation pour démentir l'unilatéralité de ces positions, mais un tel démenti ne suffit pas pour élever le point de vue jusqu'à la théologie qui, seule, est susceptible d'accorder les deux positions sans les ruiner l'une par l'autre. En mathématique ou en physique, on est fondé à retourner une proposition ou une hypothèse fausses dans leur contradictoire vraie ${ }^{9}$, alors que, en morale, il n'y a que des positions contraires qui, certes, paraissent contradictoires du point de vue humain, à savoir dans un sujet simple, mais dont l'apparente contradiction est résolue d'un point de vue extrahumain, à savoir dans un sujet double de la théologie. C'est la raison pour laquelle, l'injustice d'une maxime étant établie par l'épreuve de ses conséquences, on ne saurait rétablir pourtant la justice par l'adoption de sa contradictoire; l'homicide est injuste, mais la proscription de l'homicide n'est pas forcément juste ${ }^{10}$. On voit par là qu'est nécessaire l'élévation du point de vue produite par la théologie, qui justifie en leur ordre les deux maximes apparemment contradictoires. La vérité mathématique ou physique est simple, la vérité morale est toujours double. Et les effets anthropologiques et moraux dont le schème des "raisons des effets" ordonne graduellement les raisons ne sont pas non plus simples données dont on chercherait les causes, ce sont les figures ambivalentes d'une vérité double. Les effets répertoriés dans la liasse $\mathrm{V}$ des Pensées comportent tous raison et déraison, cette ambivalence ne trouvant de solution qu'au point haut où se dévoile la raison de la déraison.

La notion d'effet est une notion équivoque puisque, rapportée à la notion de cause, elle appartient au vocabulaire de la physique, alors que rapportée à la notion de raison, elle appartient au vocabulaire de la peinture réglée par la géométrie projective et qu'elle en vient coïncider avec la notion scripturaire de figure. On comprend que Pascal puisse parler de " raisons pourquoi figures ${ }^{11}$ ", selon une expression qui n'est évidemment pas sans évoquer celle de "raisons des effets". Faire la part du sens littéral et du sens spirituel dans l'Écriture Sainte suppose effectivement qu'on y décèle le double dessein de $D i^{12}{ }^{12}$, celui-ci voulant éclairer les uns et aveugler les autres, et ne voulant pas éclairer sans aveugler afin d'humilier ni aveugler sans éclairer afin de condamner, double dessein qui est lui-même conforme à la double capacité de tous les hommes, lesquels sont à la fois capables et indignes de Dieu $^{13}$. La Bible entière, telle une anamorphose, ne se redresse 
qu'en ce point. On peut en conclure que le schème des figuratifs n'est qu'une variante du schème des « raisons des effets ${ }^{14}$ ».

11 Le schème des "raisons des effets » traduit donc cette vérité que la vérité est double et cachée. Il ne suffit pas de dire vrai sans apercevoir en quoi ce qu'on dit est vrai ou sans démêler le vrai du faux dans ce qu'on dit. La vérité n'est pas hors des opinions, elle est dans les opinions communes, mais « sans différence à l'extérieur ${ }^{15}$ ». Ainsi, le peuple a des opinions très saines, mais elles ne le sont pas dans sa tête, comme le note Pascal, parce qu'il pense que la vérité est où elle n'est pas. Il se méprend sur le lieu de la vérité : « $\mathrm{La}$ vérité est bien dans leurs opinions, mais non pas au point où ils se figurent ${ }^{16}$.» Il faut dire par conséquent la même chose que le peuple et professer les mêmes opinions que lui, quoique par une autre pensée que lui.

La liasse $\mathrm{V}$ des Pensées a pour fonction de rendre raison de ce qui est apparemment déraisonnable, en particulier de fonder l'obéissance civile à un ordre civil pourtant luimême dénué de fondement. Mais, précisément parce que le schème des «raisons des effets » hausse la justification jusqu'à la vraie raison de l'obéissance civile, il en indique aussi les limites. Le point de vue des chrétiens parfaits intègre le point de vue du peuple et sa revendication d'une pure justice. Il n'est pas injustifié de viser, au-delà du point de vue des habiles, autre chose que l'ordre. Montaigne a tort ${ }^{17}$ de ne pas prendre en compte la requête de justice qui produit l'illusion du peuple. Le peuple a certes tort, mais il a aussi raison, et c'est ce que les habiles ne voient pas, qui veulent qu'on obéisse aux lois par cette seule raison qu'elles sont lois, les hommes étant naturellement incapables de justice. On ne pourra supprimer l'exigence de justice, même si cette exigence demeure naturellement vide. Que les hommes soient naturellement incapables d'assigner le juste ne les empêche pas d'y aspirer ${ }^{18}$. Ils ne veulent s'obliger qu'envers la justice et il serait tyrannique et vain de prétendre les en détourner ${ }^{19}$. Car, replacée en son ordre, cette exigence de justice est tout à fait justifiée. Se situer au point de vue des chrétiens parfaits revient donc à justifier l'ordre en le limitant: car l'ordre n'est lui-même justifié que replacé également en son ordre. Et la justice, et l'ordre sont ainsi ramenés à leur ordre propre. Le point de vue des chrétiens parfaits ordonne différents ordres qui sont irréductibles les uns aux autres et dont il convient de respecter la spécificité.

On appréciera sans peine la portée de ces analyses pour démontrer que Pascal admet un droit, et même un devoir, de résistance ${ }^{20}$. La politique ayant été assignée à son ordre propre, on distinguera deux transgressions possibles de l'ordre. La première, formelle, consiste à abolir la différence des ordres : c'est le cas lorsque le pouvoir politique prétend se substituer au pouvoir religieux et qu'il en usurpe les prérogatives (ou l'inverse, lorsqu'il s'agit des dévots). La seconde, matérielle, consiste à éluder les implications de la spécificité de chaque ordre : c'est le cas lorsque, sous prétexte que les lois ne coïncident jamais avec la pure justice, le pouvoir politique s'arroge le droit de promulguer les décrets injustes. Déférer à un pouvoir dont les lois assurent l'ordre et le salut public n'est pas déférer à un pouvoir arbitraire dont les décrets compromettent au contraire l'ordre et le salut public. La liasse V des Pensées corrobore la XIVème Provinciales. Parler de lois injustes signifie seulement qu'elles ne sont pas justes, cela n'empêche pas qu'elles ne doivent demeurer lois. Elles ne sont pas justes, car elles n'ont pas pour fin la justice mais l'ordre. Cependant, elles ne sauraient évacuer toute justice. Car, bien que l'homme pécheur ne sache plus assigner le juste, il reste néanmoins capable de dénoncer l'injustice en faisant l'épreuve des conséquences qui lui sont attachées. Aussi les lois n'ont-elles nullement pour fin de mettre fin à l'exigence de justice, mais elles la contiennent dans la 
seule forme appropriée à l'injustice du péché, à savoir l'ordre civil. C'est en le comprenant, qu'on comprend que l'ordre civil soit déclaré une image ou un tableau de l'ordre religieux commandé par la charitée ${ }^{21}$.

Il convient de montrer à présent comment la conjonction de l'Entretien avec M. de Sacy et des Écrits sur la grâce permet d'étendre à la théologie le discernement critique qu'ellemême est censée rendre possible dans la liasse V des Pensées.

On a indiqué précédemment qu'il ne suffisait pas de confronter avec la condition de l'homme les hypothèses explicatives formulées par Épictète et Montaigne afin de démentir l'unilatéralité de ces positions, car un tel démenti de part et d'autre - la faiblesse infirmant Épictète et la grandeur infirmant Montaigne - n'élevait pas encore le point de vue jusqu'à la théologie qui, seule, pouvait concilier les deux positions sans les détruire l'une par l'autre. Tant qu'on en reste à la perspective purement humaine qui est celle de la philosophie, le double démenti opposé à Épictète et à Montaigne ne mène qu'à la destruction mutuelle de leurs deux positions, grandeur et faiblesse ne pouvant se conjuguer dans un sujet simple. L'Entretien bute sur ce dilemme: aucune des deux positions ne peut subsister seule, étant démentie dans son unilatéralité, et elles ne peuvent davantage subsister toutes les deux ensemble, puisqu'elles présentent alors une contradiction insoluble du point de vue humain. Il faut donc recourir à la théologie, afin de distribuer sur deux sujets en l'homme la grandeur et la faiblesse que la philosophie ne saurait unir. La doctrine augustinienne des deux états ou des deux natures rapporte ainsi la grandeur à la création divine et la faiblesse à la corruption humaine, la grandeur à la grâce qui hausse l'homme au-dessus de la nature, la faiblesse au péché qui dégrade l'homme au-dessous de la nature. L'homme actuel, capable et indigne de Dieu, donne donc à la fois raison et tort, et à Épictète, qui n'a reconnu que sa grandeur dans ses devoirs, et à Montaigne qui, inversement, n'a reconnu que sa faiblesse dans son impuissance. Cette vérité double, la théologie l'enseigne et l'incarnation la réalise ${ }^{22}$, de sorte que les positions nécessairement duelles de la philosophie en sont des figurations. Il y a dans chacune d'elles quelque conformité avec la sagesse véritable, qui la fait réputer image ou figure. Car ce n'est pas seulement la nature qui peint Dieu en ses ouvrages, lesquels portent tous l'empreinte de sa double infinité et de la toute-puissance divine dont cette double infinité est le caractère sensible, c'est surtout l'esprit qui peint Dieu dans sa recherche du vrai et qui exprime le vrai jusque dans le refoulement dont témoignent ses erreurs ${ }^{23}$. Le vrai ne s'affirme pas directement dans ce qu'affirment Épictète et Montaigne, le vrai s'affirme au rebours de ce qu'ils affirment, il affirme dans ce qu'ils nient en l'excluant. L'homme étant misérable - n'étant grand que dans sa faiblesse et n'étant faible que dans sa grandeur -, chaque affirmation n'est vraie que dans l'affirmation contraire. Tandis que la nature peint Dieu de manière directe et statique, l'esprit peint Dieu de manière indirecte et dynamique.

16 Cependant, si la théologie s'avère la vérité de la philosophie dans ses efforts pour atteindre la sagesse véritable, comme c'est le cas dans l'Entretien avec M. de Sacy, comment comprendre qu'elle-même fasse l'objet, dans les Écrits sur la grâce cette fois, d'un discernement qu'elle semble seule apte à effectuer? Pourquoi Pascal ne se contente-t-il pas d'appliquer aux apories de la philosophie les ressources surnaturelles que lui procure l'orthodoxie augustinienne transmise par l'Église? On imagine un second M. de Sacy, s'étonnant que l'exposé de l'orthodoxie, telle qu'elle a été réaffirmée au concile de Trente en bannissant par anathèmes les erreurs des molinistes et des protestants, s'accompagne ici de l'éclaircissement de ces erreurs que Pascal, quant à lui, juge indispensable pour 
faire ressortir la vérité de l'augustinisme. Il ne suffit donc pas de livrer le contenu vrai de la théologie, il faut montrer en outre comment les erreurs concourent à sa détermination et comment elles sont, en retour, jugées par lui. Mais n'est-ce pas à la philosophie qu'incombe cette tâche de subsomption des erreurs sous la vérité ? Si l'orthodoxie est fondée et déterminée, dans son contenu, par l'autorité religieuse, ne revient-il pas néanmoins à la philosophie de fonder et de déterminer une nouvelle fois ce contenu vrai par sa réduplication dans la forme vraie du schème des « raisons des effets » qui, loin de faire rejeter les erreurs, permet au contraire de les intégrer comme autant de figures de la vérité? On verra ensuite quelles sont les conséquences, quant à la question de l'obéissance religieuse, de cette reprise philosophique de la théologie.

Alors que l'ordre civil est dénué de fondement, car il n'est pas fondé sur la justice mais sur l'institutionnalisation de la force, l'ordre religieux est, quant à lui, très bien fondé sur Jésus-Christ et sur la tradition de l'Église apostolique. L'obéissance civile n'est pas fondée dans l'ordre civil lui-même, mais elle est fondée dans un point de vue extrapolitique, dans le point de vue théologique qui perçoit en cet ordre une image ou un tableau de celui que produit la charité. L'obéissance religieuse, en revanche, est susceptible d'être fondée dans l'ordre religieux lui-même. Il suffit que l'Église se prononce pour que l'obéissance à ses décisions soit requise. Mais encore faut-il se souvenir qu'il y a une genèse historique de la vérité en théologie. La vérité portée par l'Église, que la théologie a la vocation d'éclaircir, est tout entière contenue en germe dans le Christ. Pascal le dit bien à la fin de l'Entretien, Jésus-Christ est le modèle incarnant toutes les apparentes contradictions sur lesquelles la philosophie achoppe et dont seule la théologie lui apporte la solution. Mais la vérité qui est en germe dans le Christ doit être développée dans son Église. Et on sait que ce sont les hérésies à combattre qui en offrent l'occasion. Les hérésies se déclarant, l'Église approfondit sa doctrine pour y répondre ${ }^{24}$. La réponse est nécessaire dans son contenu en ce sens qu'elle est conforme au fondement: ni les Pères, ni les Papes, ni les conciles ne peuvent contredire ce qui a préalablement été posé en Jésus-Christ, ils ne peuvent qu'en déployer toutes les incidences. Cependant, cette réponse nécessaire dans son contenu ne laisse pas de rester contingente dans sa forme, puisque c'est de l'extérieur, à l'occasion des hérésies, que cette réponse est proposée. Or il y a une grande différence entre dire les choses par rencontre, l'occasion s'en présentant alors qu'elle pouvait aussi bien ne pas se présenter, quoique, le principe étant ferme, il soit sûr que, l'occasion s'en présentant en effet, on ne peut pas ne pas dire ces choses ${ }^{25}$, et dire les choses non pas par rencontre, mais par principe, en substituant à la forme de la contingence historique la forme de la systématicité logique. La fin de l'opuscule De l'art de persuader contient même une version originale du schème des "raisons des effets »: on peut dire les mêmes choses 1) par hasard, 2) avec un principe quoique par rencontre (ce qui caractérise le discours théologique), 3) par principe et en les articulant de manière systématique à partir d'un principe alors pris pour fondement (ce qui caractérise, avec Descartes, le discours philosophique). N'est-ce pas précisément ce que Pascal tente dans les Écrits sur la grâce, en considérant les erreurs théologiques non plus comme des accidents qui n'affecteraient pas le vrai de l'intérieur, mais comme des figures dont l'articulation marque le lieu du vrai à l'intérieur même du Vrai? Pascal se rallie indéniablement au discours philosophique. Cependant, le principe de Descartes est interne à la philosophie, alors que le principe de Pascal lui est externe. La philosophie ne possède pas en elle-même son propre principe, c'est dans la théologie qu'elle le trouve mais, celui-ci étant posé, il devient pour elle le principe d'une articulation systématique et d'elle-même, et de la 
théologie. On n’hésitera donc pas à qualifier de philosophique la théologie des Écrits sur la grâce.

18 La mise en forme triadique adopté par Pascal n'est pas simple procédé pédagogique, elle est la forme dans laquelle ressort la vérité du contenu. Il ne s'agit pas, pour la philosophie, de comprendre le mystère fondamental de la foi - l'incarnation - que la théologie approfondit sous l'impulsion des hérésies, mais il s'agit pour elle d'appliquer ce mystère fondamental, en tant qu'il a été reconnu par elle raison de toutes $\operatorname{choses}^{26}$, à la compréhension de la vérité et de l'erreur inséparables en toutes choses, même en théologie. La philosophie ne s'approprie pas le contenu des dogmes chrétiens, mais il lui revient de départager la vérité de l'erreur à la lumière de leur principe fondamental. L'augustinisme, conforme à Jésus-Christ par la doctrine des deux états ou des deux natures en l'homme, redresse la vérité jusque dans l'erreur, celle-ci consistant ici à méconnaître un des deux états ou une des deux natures en l'homme. C'est ainsi que les molinistes étendent à la seconde nature ce qui ne vaut que pour la première - la volonté divine de sauver tous les hommes et le caractère suffisant de la grâce -, tandis que les protestants étendent à la première nature ce qui ne vaut que pour la seconde - l'arbitraire de la volonté divine et le caractère efficace de la grâce poussé jusqu'à la suppression du libre arbitre -, extension qui, effectuée en ce sens, fausse complètement et la première, et la seconde nature, puisque la seconde nature succède à la première nature, et non l'inverse. Si Pascal déclare les thèses protestantes entièrement fausses, ce n'est donc pas qu'il leur dénie de figurer le vrai en quelque façon. Comme les molinistes, les protestants ne font que déformer sans cesser de la figurer la doctrine augustinienne des deux états ou des deux natures en l'homme. Et ce complet renversement de la vérité, qui la figure encore jusque dans la déformation qu'elle lui fait subir, son origine n'étant que dans l'omission d'un de ses deux aspects, culmine logiquement dans la violation de la charité par laquelle les protestants, se séparant de l'Église, rompent son unité. Le schème des « raisons des effets» justifie la correspondance de la vérité et de la charité : on est d'autant plus charitable qu'on connait la part de vérité présente en toutes choses, et on l'est d'autant moins qu'on ignore le degré d'éloignement du vrai où on est tombé. Il est par conséquent possible d'aller jusqu'à l'explication de la dissymétrie dans la symétrie des erreurs. L'erreur n'apparaît plus comme contingente, elle apparaît comme nécessaire. La philosophie justifie après coup, par la mise en forme qu'elle lui donne, le procès de détermination de la vérité qui s'accomplit historiquement dans l'Église. Les Écrits sur la grâce ne relèvent pas seulement la cohérence des auteurs orthodoxes jusque dans la collection de leurs œuvres - à l'instar des auteurs de la Bible, ils forment pour ainsi dire un seul auteur-, mais ils confèrent de surcroît à cette cohérence théologique une systématicité philosophique.

19 Les Pensées contiennent plusieurs fragments qui prolongent ces réflexions. La vérité qu'enseigne la théologie étant double, les erreurs sont également duelles et elles revêtent toujours la même forme quel qu'en soit le contenu: qu'il s'agisse de la liberté et de la grâce de la prédestination, de Jésus-Christ, de l'Église, du pape et des conciles, de l'interprétation de l'Écriture, des sacrements, de l'eucharistie en particulier, l'orthodoxie est suspendue entre deux hétérodoxies contraires dont les attaques conjuguées n'aboutissent qu'à leur destruction mutuelle et qui concourent ainsi au renforcement de la vérité contre laquelle elles prétendent s'établir ${ }^{27}$. L'orthodoxie sort victorieuse des controverses, parce que sa vérité n'est pas sujette à la controverse, mais au discernement. 
20 La leçon qu'on doit tirer de l'Entretien avec M. de Sacy est que la théologie peut bien éclairer la philosophie sans en rien vouloir savoir et que c'est donc encore à la philosophie d'éclairer la théologie sur les lumières que celle-ci dispense. Avec les Écrits sur la grâce, cette leçon se répète dans la théologie elle-même, car l'orthodoxie peut bien pareillement repousser les hérésies sans vouloir savoir en quoi elle les redresse. Mais, en dehors du jugement par lequel sont appliquées les lumières de la théologie et de l'orthodoxie, la soumission, pourtant due à celle-ci du seul fait de leur fondement, peutelle être elle-même entièrement fondée ? La liasse $\mathrm{V}$ des Pensées montre que l'obéissance civile n'est pas fondée en dehors du point de vue de la théologie. Il ne suffit pas de dire qu'il faut obéir aux lois, il faut le dire en vérité et localiser le vrai dans le vrai. Le lieu du vrai désigne le lieu de la soumission, car la soumission doit être éclairée dans sa forme comme dans son contenu. On ne saurait se contenter de se soumettre, sans savoir à quoi et pourquoi on doit se soumettre, Mais, savoir où on doit se soumettre, c'est ne se soumettre que là où on juge qu'on doit se soumettre. Cependant, quand on appréhende ainsi le lieu de la soumission, on appréhende aussi les limites de la soumission. Effectivement, l'obéissance civile n'est fondée que pour autant que l'ordre civil se cantonne dans son ordre, qui n'est ni celui de la pure justice, ni celui de la pure force. On aura le droit et le devoir de dénoncer un ordre civil tyrannique. Mais n'en va-t-il pas de même pour la soumission due à la théologie ?

On a vu à propos de la politique qu'il ne fallait obéir qu'en son ordre. Mais ne serait-il pas tyrannique que la théologie en vînt à s'attribuer le monopole du vrai sous prétexte que c'est en elle qu'on découvre la vérité de toutes les vérités ? Déclarer, comme le fait Pascal, que la théologie est le centre de toutes les sciences ${ }^{28}$, ne signifie évidemment pas que toute science soit réductible à la théologie. Ce n'est pas parce que la théologie s'avère la condition (formelle) de la détermination d'un ordre propre du politique, qu'il lui revient de déterminer et de conditionner matériellement et en son contenu l'ordre propre du politique. Au contraire, il faut récuser la tyrannie des dévots qui croient pouvoir appliquer autrement que formellement les lumières que la théologie leur donne et qui prétendent dicter ses lois à l'ordre politique ${ }^{29}$. Il faut récuser de manière similaire la tyrannie du pape lorsque celui-ci méconnaît l'ordre propre de la science et qu'il décide de la vérité en physique, ou lorsqu'il confond matières de droit et matières de fait et qu'il impose une signature où le droit est censé emporter le fait ${ }^{30}$. La théologie ordonne formellement tous les autres ordres, mais elle ne s'en subordonne pas le contenu. Elle est, à l'inverse, le principe même de la différenciation des ordres. Aussi la soumission à la théologie n'est-elle pas entièrement fondée en dehors du jugement et de l'acte philosophique qui ne soumettent pas à la théologie sans lui faire rendre à chaque ordre sa propriété.

Il y a un ordre propre du politique, identifiable quand on voit, à la lumière de la théologie, ce qui fonde l'obéissance civile à un ordre civil sans fondement. On confessera alors que les rois n'exercent leur empire que dans l'ordre des corps, qu'ils sont rois de concupiscence, et que le propre de leur puissance en cet ordre, est de protéger et de faire des heureux ${ }^{31}$. De même, il y a un ordre propre de la science, identifiable quand on voit, à la lumière de la théologie, ce qui fonde l'investigation d'une nature avec laquelle l'homme n'a pourtant aucune proportion ou ce qui fonde le progrès d'une connaissance pourtant sans fondement ${ }^{32}$. Car on ne peut pas plus renoncer au vrai qu'on ne peut renoncer au juste, mais cette exigence de vérité sera contenue dans l'ordre scientifique - et articulée au pyrrhonisme - comme l'exigence de justice est contenue dans l'ordre politique - et 
articulée aussi au pyrrhonisme : et le pyrrhonisme, et la science sont justifiés, mais replacés en leur ordre, à partir du point de vue de la théologie qui ne requiert pas la soumission sans inclure le jugement ${ }^{33}$. On avouera alors que les savants n'exercent leur empire que dans l'ordre des esprits et que le propre de leur puissance, en cet ordre, est de satisfaire la curiosité. Mais, en assignant ainsi à la science son ordre propre, on mesure la relativité de son savoir et on évite de la confondre avec la sagesse. La science, qui ne connaît jamais qu'un milieu des choses, se parfait dans la docte ignorance ${ }^{34}$. Le point de vue adopté ici condamne définitivement l'entreprise cartésienne. Si Descartes a pu être gratifié d'un esprit de discernement, dans l'opuscule De l'art de persuader, pour avoir élevé au rang de principe métaphysique le cogito que saint Augustin avait formulé seulement par rencontre, il ne l'a été qu'au plan formel, pour avoir promu un type de discours - le discours philosophique - qui, par sa systématicité, dépasse la cohérence du discours théologique. Descartes est un philosophe, mais sa philosophie est fausse. On peut à présent lui retirer l'esprit de discernement dont on l'avait d'abord gratifié, pour avoir ignoré, en prétendant déduire une physique entière de ce principe métaphysique, l'ordre propre de la science. Il faut certes professer le mécanisme physique, mais sans avoir l'ambition de constituer un système de physique ${ }^{35}$. La gradation des trois ordres, assortie de la gradation des trois concupiscences, exclut Descartes ${ }^{36}$.

Peut-être se trouve maintenant résolu le problème de la conciliation de la structure horizontale et de la structure verticale des ordres. Il y a, de fait, différents ordres, et différents règnes, et sentiment d'injustice quand on est confronté au désir de domination universel hors de tout ordre ${ }^{37}$. Mais ce n'est qu'à partir d'un ordre dominant parce que discriminant, celui de la théologie, qu'on peut discerner la propriété de chaque ordre et passer ainsi, d'une diversité de fait et d'un sentiment d'injustice. L'esprit de discernement suppose l'élévation du point de vue jusqu'à celui qui embrasse toutes les vérités en leur ordre. L'appréhension de la "justice interne» des ordres est subordonnée à l'appréhension de leur «justice externe» ou de leur gradation. "Se moquer de la philosophie » en se moquant de sa partialité ne conduit pas à abandonner tout jugement et toute philosophie pour s'abandonner à la soumission et à la théologie, puisque, au contraire, «c'est [là] vraiment philosopher ${ }^{38}$ ". Le philosopher relaie la philosophie, celle de Pascal consistant à éclairer tout à la lumière de la théologie et à éclairer la théologie elle-même sur sa dimension philosophique.

\section{NOTES}

1. Le mathématicien Girard Desargues (1591-1661) a inspiré l'Essai pour les coniques et la Generatio conisectionum de Pascal. Mais l'influence d'Abraham Bosse (1602-1676) est décelable dans les Pensées où le modèle de la géométrie projective est enveloppé dans les images picturales dont Pascal se sert pour constituer son anthropologie, puis son herméneutique.

2. Il s'agit du titre d'un ouvrage d'A. Bosse. En ce qui concerne son œuvre écrite, voir Le peintre converty aux règles de son art, Paris, Hermann, coll. « Miroirs de l'art », 1964, p. 187-189.

3. Voir notamment Laf. 130, Br. 420 et Laf. 400. Br. 427. Sur l'excès et le défaut, voir Laf. 21, Br. 381, Laf. 38, Br. 71, Laf. 41, Br. 69, Laf. 57, Br. 379, Laf. 183, Br. 253, Laf. 199, Br. 72, Laf. 252, 
Br. 648, Laf. 518, Br. 378, Laf. 519, Br. 70, Laf. 674, Br. 359, Laf. 681, Br. 353, Laf. 723, Br. 69, Laf. 783, Br. 357.

4. Voir Laf. 90, Br. 337 et Laf. 93, Br. 328.

5. Voir Laf. 14, Br. 338.

6. On suivra ainsi de préférence Michel Serres, Le système de Leibniz et ses modèles mathématiques, Paris, P.U.F., 1968, en 2 volumes.

Ce développement est à confronter avec celui de Laurent Thirouin, dans « Raison des effets, essai d'explication d'un concept pascalien ", revue XVII siècle, $34^{\mathrm{e}}$ année ( $\left.{ }^{\circ} 1\right), \mathrm{n}^{\circ} 134$, janv.-mars 1982 , p. 31-50. Sur le schème physique, L. Thirouin renvoie lui-même à Jean Molino, «La raison des effets ", in Méthodes chez Pascal, Paris, P.U.F, 1979, p. 477-493.

7. Voir lettre au P. Noël du 29 octobre 1647, in Cuvres complètes, Paris, Seuil, coll. « L'Intégrale », 1963, p. 202 (b).

8. Voir préface au Traité du vide.

9. Pour les mathématiques, voir la réfutation par l'absurde de la thèse du chevalier de Méré, dans l'opuscule De l'esprit géométrique; pour la physique, voir la méthode d'établissement de l'hypothèse vraie par l'infirmation expérimentale de sa contradictoire (supra, note 7).

10. Voir Laf. 729, Br. 931 et Laf. 905, Br. 385.

11. Laf. 502, Br. 571.

12. Voir la liasse XVIII, en particulier Laf. 232, Br. 566 et Laf. 236, Br. 578.

13. Dans la liasse XVIII, voir Laf. 239, Br. 510. Voir aussi Laf. 444, Br. 557 et Laf. 449, Br. 556.

14. Voir Laf. 223, Br. 570 qui ouvre la liasse XVIII : les considérations sur la cause des figures sont à mettre au chapitre des fondements.

15. Laf. 225, Br. 789.

16. Laf. 92, Br. 335.

17. Voir Laf. 525, Br. 325.

18. Voir Laf. 131, Br. 434, Laf. 401, Br. 437 et Laf. 406, Br. 395.

19. Voir Laf. 525, Br. 325 : « car on ne veut être assujetti qu'à la raison ou à la justice. La coutume sans cela passerait pour tyrannie, mais l'empire de la raison et de la justice n'est non plus tyrannique que celui de la délectation. Ce sont les principes naturels à l'homme. »

20. Contre la thèse soutenue par Christian Lazzeri dans Force et justice dans la politique de Pascal, Paris, P.U.F., coll. « Philosophie d'aujourd'hui », 1993.

21. Voir Laf. 106, Br. 403 et Laf. 118, Br. 402. Voir aussi Laf. 210, Br. 451 et Laf. 211, Br. 453.

22. Voir Entretien avec M. de Sacy, O.C., p. 296 (b) : „ C'est donc de ces lumières imparfaites qu'il arrive que l'un, connaissant les devoirs de l'homme et ignorant son impuissance, se perd dans la présomption, et que l'autre, connaissant l'impuissance et non le devoir, il s'abat dans la lâcheté ; d'où il semble que, puisque l'un conduit à la vérité, l'autre à l'erreur, l'on formerait en les alliant une morale parfaite. Mais, au lieu de cette paix, il ne résulterait de leur assemblage qu'une guerre et qu'une destruction générale : car l'un établissant la certitude, l'autre le doute, l'un la grandeur de l'homme, l'autre sa faiblesse, ils ruinent la vérité aussi bien que les faussetés l'un de l'autre. De sorte qu'ils ne peuvent subsister seuls à cause de leurs défauts, ni s'unir à cause de leurs oppositions, et qu'ainsi ils se brisent et s'anéantissent pour faire place à la vérité de l'Évangile. C'est elle qui accorde les contrariétés par un art tout divin, et, unissant tout ce qui est de vrai et chassant tout ce qui est de faux, elle en fait une sagesse véritablement céleste où s'accordent ces opposés, qui étaient incompatibles dans ces doctrines humaines. Et la raison en est que ces sages du monde placent les contraires dans un même sujet; car l'un attribuait la grandeur à la nature et l'autre la faiblesse à cette même nature, ce qui ne pouvait subsister; au lieu que la foi nous apprend à les mettre en des sujets différents : tout ce qu'il y a d'infirme appartenant à la nature, tout ce qu'il y a de puissant appartenant à la grâce. Voilà l'union étonnante et nouvelle que Dieu seul pouvait enseigner, et que lui seul pouvait faire, et qui n'est qu'une image et qu'un effet de l'union ineffable de deux natures dans la seule personne d'un Homme-Dieu. » 
23. Voir ibid., O. C., p. 296 (a) : "J'ai pris un plaisir extrême à remarquer dans ces divers raisonnements en quoi les uns et les autres sont arrivés à quelque conformité avec la sagesse véritable qu'ils ont essayé de connaître. Car, s'il est agréable d'observer dans la nature le désir qu'elle a de peindre Dieu dans tous ses ouvrages, où l'on en voit quelque caractère parce qu'ils en sont les images, combien est-il plus juste de considérer dans les productions des esprits les efforts qu'ils font pour imiter la vertu essentielle, même en la fuyant, et de remarquer en quoi ils y arrivent et en quoi ils s'en égarent, comme j'ai tâché de faire dans cette étude ! »

24. Voir Laf. 313, Br. 569 et Laf. 536, Br. 579.

L'histoire de l'Église est l'histoire de la vérité (voir Laf. 776, Br. 858) parce que le triomphe de la vérité dont l'Église est dépositaire est assuré par le heurt des hérésies contraires (voir Laf. 743, Br. 859 et Laf. 758, Br. 857) :

«L'Église a toujours été combattue par des erreurs contraires. Mais peut-être jamais en même temps comme à présent, si elle en souffre plus à cause de la multiplicité d'erreurs, elle en reçoit cet avantage qu'ils se détruisent. » (Laf. 733, Br. 862) Voir aussi I Ir Écrit sur la grâce, O.C., p. 313 (b) 314 (a).

25. Voir Laf. 930, Br. 513.

26. Voir Laf. 449, Br. 556 : «Qui le connaît (Jésus-Christ) connaît la raison de toutes choses. » 27. La vérité est double : voir Laf. 443, Br. 863, Laf. 576, Br. 567, Laf. 733, Br. 862, Laf. 786, Br. 865. Pour les applications, voir Laf. 567, Br. 874, Laf. 569, Br. 872, Laf. 604, Br. 871, Laf. 706, Br. 870, Laf. 726, Br. 876, Laf. 867, Br. 875, Laf. 874, Br. 881 ; Laf. 571, Br. 775, Laf. 733, Br. 862, Laf. 760, Br. 568, Laf. 774, Br. 497, Laf. 791, Br. 777, Laf. 712, Br. 530, Laf. 785, Br. 776, Laf. 910-911-912, Br. 781.

28. Voir Laf. 65, Br. 115.

29. Voir Laf. 90, Br. 337.

30. Voir XVII ${ }^{\text {ème }}$ et XVIII ${ }^{e ̀ m e}$ Provinciales. Voir aussi Écrit sur la signature du formulaire.

31. Voir trois Discours sur la condition des Grands. Voir aussi Laf. 796, Br. 314 et Laf. 797, Br. 310.

32. Voir Laf. 199, Br. 72 reprenant la préface au Traité du vide.

33. Voir Laf. 170, Br. 268.

34. Voir Laf. 83, Br. 327. En y joignant Laf. 82, Br. 271 et Laf. 84, Br. 79, on a trois fragments sur la science et la sagesse dont l'inclusion dans la liasse $\mathrm{V}$ est parfaitement justifiée.

35. Voir Laf. 84, Br. 79 et Laf. 553, Br. 76.

36. Voir Laf. 308, Br. 793 et Laf. 933, Br. 460.

37. Voir Laf. 58, Br. 332.

38. Laf. 513, Br. 4.

INDEX

Mots-clés : Pascal, raison des effets, théologie, philosophie

Keywords : reason, effect, theology, philosophy

\section{AUTEUR}

HÉLÈNE BOUCHILLOUX

Département de philosophie, Université de Lorraine 